\title{
Guías de práctica clínica y otros productos basados en la evidencia, en la consulta
}

\author{
Jl. Martín Sánchez, JM. Mengual Gil \\ Instituto Aragonés de Ciencias de la Salud. Secretaría de GuíaSalud, \\ Biblioteca de Guías de Práctica Clínica del Sistema Nacional de Salud. Zaragoza. España
}

Rev Pediatr Aten Primaria. 2009; I I (Supl I7):s457-s463

Juan Ignacio Martín Sánchez, jimartin.iacs@aragon.es

Resumen

GuíaSalud es un organismo del Sistema Nacional de Salud (SNS) en el que participa el conjunto de comunidades autónomas (CC. AA.), que cuenta con el refrendo del Consejo Interterritorial (CI) y el apoyo del Ministerio de Sanidad y Política Social a través de la Agencia de Calidad del SNS. Desde que da su primeros pasos, a mediados del año 2002, hasta la actualidad, se ha visto inmerso en cambios enmarcados en el Plan de Calidad del SNS, siendo el último, descrito en el Plan de Calidad del año 2007 el que implica un giro importante en objetivos, introduciéndose en la cartera de trabajo el desarrollo de productos basados en la evidencia científica que ayuden a los profesionales sanitarios en la toma de decisiones.

Esto implica un cambio en la estructura organizativa de dirección y gestión de GuíaSalud, dedicación de recursos dirigidos a fortalecer el desarrollo de guías de práctica clínica (GPC) en nuestro país, y a abrir nuevas líneas de trabajo encaminadas a enriquecer la oferta de productos a los profesionales sanitarios, como es el caso para las preguntas clínicas.

En esta estrategia se cuenta con las personas que integran el Comité Científico de GuíaSalud, su Consejo Ejecutivo (CE), y aquellos profesionales sanitarios interesados en una metodología de trabajo sistemática y explícita y que constituyen la red de colaboración de GuíaSalud. Desde la Secretaría de GuíaSalud se cuenta con un equipo de profesionales conocedores de la metodología de trabajo adecuada y una web 2.0 dirigida a interaccionar con los profesionales sanitarios.

Palabras clave: Guía de práctica clínica, Medicina basada en la evidencia, Red de colaboración.

Abstract

GuíaSalud is a National Health System (NHS) agency, which includes all the Autonomous Communities (CCAA), and has the endorsement of the inter-territorial council (IC) which is supported by the Ministry of Health and Social Policy through the NHS Quality Agency. Since its first steps, in mid-2002 until now, it has been immersed in framework changes within the quality of the NHS Plan. The latter being described in the quality plan of 2007, which involves important changes in the objectives, introducing into the work portfo-

Los autores declaran no presentar posibles conflictos de intereses en relación con la preparación y publicación de este artículo. 
lio the development of products, based on scientific evidence to assist health professionals in decision-making.

This involves changes in the organizational structure of leadership and management of GuíaSalud, dedication of resources aimed at strengthening the development of clinical practice guidelines (CPG) in our country, and open new lines of work aimed at enrichment the offer of products to the health professionals, as is the case for clinical questions.

This strategy includes professionals who are part of GuiaSalud's Scientific Committee, Executive Council (EC), and other health professionals interested in working with a systematic and explicit methodology; and are part of the GuíaSalud collaborative network. The GuíaSalud department has a team of professionals who know about the appropriate methodology and a web 2.0 designed to interact with health professionals.

Key words: Practice guideline, Evidence-based medicine, Collaborative network.

\section{Introducción}

GuíaSalud es un organismo del SNS en el que participan todas las comunidades autónomas (CC. AA). Surge a mediados del año 2002 por iniciativa de la Comunidad Autónoma de Aragón y de la Fundación Salud Innovación y Sociedad. En noviembre de 2002, el Ministerio de Sanidad y Consumo dio su apoyo explícito al proyecto y propone que se presente en el seno del Consejo Interterritorial (CI). Con fecha 26 de marzo de 2003, el proyecto es refrendado por el $\mathrm{Cl}$, como elemento de apoyo y mejora de la asistencia sanitaria en el Sistema Nacional de Salud (SNS).

En octubre de 2003, el Instituto Aragonés de Ciencias de la Salud (I+CS), que asume la coordinación del proyecto, firma un convenio con el Ministerio de Sanidad y Consumo. En ese convenio se estableció que el I+CS, como unidad de gestión, es el responsable del desarrollo, gestión y seguimiento del proyecto y que además actúa como interlocutor con los responsables de las CC. AA. y de la Agencia de Calidad del SNS, en todos los aspectos técnicos y de gestión necesarios para la consolidación del Proyecto GuíaSalud. En abril de 2005 presentó su Portal GuíaSalud (www.guiasalud.es)'

En 2006, el Plan de Calidad del SNS plantea, en su estrategia para mejorar la práctica clínica, consolidar y extender el Proyecto GuíaSalud. Además, encarga el desarrollo de una metodología común para la elaboración de guías de práctica clínica $(G P C)^{2}$ y la realización de GPC que aborden temáticas de las líneas estratégicas del Plan Nacional de Calidad. En 2007, el Ministerio de Sanidad y Consumo promueve la creación de una Biblioteca de GPC del SNS cuya dirección recae en GuíaSalud.

Antes esa situación, en la reunión del Consejo Ejecutivo (CE), realizada en San- 
tander en febrero de 2007, se vio necesario definir y analizar qué actuaciones se deben desarrollar para ampliar los recursos y servicios de GuíaSalud y de esa forma potenciar su papel en el SNS. Como consecuencia de ese trabajo se identificaron una serie de acciones, sobre financiación, implementación, gestión, sistemas de producción y metodología, productos, etc., que circularon entre todos los miembros del CE y que sirvieron de base para estructurar la propuesta de trabajo, que se presentó en la reunión del CE realizada en Toledo en septiembre de 2007.

GuíaSalud-Biblioteca de GPC en el SNS tiene por misión, en esta nueva eta- pa, potenciar la oferta y utilización de recursos, servicios y productos basados en la evidencia científica para apoyar la toma de decisión de los profesionales y de los pacientes en el SNS, así como impulsar la red de colaboración entre entidades y profesionales relacionados con las GPC y la medicina basada en la evidencia (MBE).

\section{Material y métodos}

Se articula una nueva estructura organizativa de dirección y gestión (figura 1). El período de tiempo que abarca el presente trabajo va desde el año 2006 al presente.

\section{Figura 1. Estructura organizativa de GuíaSalud-Biblioteca de GPC del SNS.}

\section{gu@̊asalud}

\section{Estructura organizativa}

\section{Consejo interterritorial}
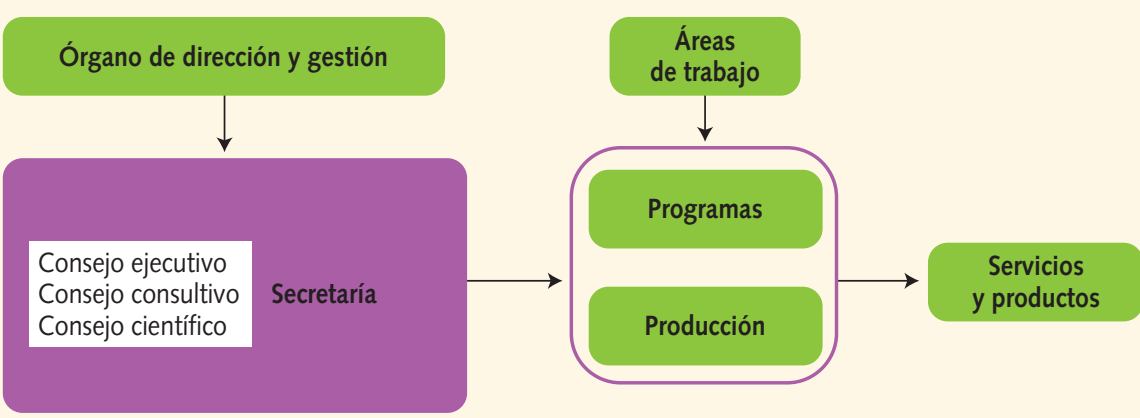
Los objetivos que este nuevo órgano se plantea son:

- Promover la elaboración, adaptación y actualización de GPC y otros instrumentos basados en la evidencia científica, acordes a las necesidades del SNS y relevantes para el trabajo de los profesionales sanitarios.

- Facilitar el acceso y la utilización de un número significativo de guías y otros instrumentos y recursos basados en la evidencia para el apoyo a la toma de decisiones de los profesionales.

- Favorecer la implementación y utilización de las GPC.

- Evaluar el impacto en salud y la mejora continua de la calidad en relación a la aplicación de las GPC en el SNS.

- Promover la formación para la elaboración, implementación y evaluación del impacto de las GPC y desarrollar herramientas de ayuda a los profesionales sanitarios.

- Promover la investigación en el ámbito de las GPC.

Para dar respuesta a estos objetivos en el horizonte temporal, se marcan unas áreas de trabajo, las cuales, en su desarrollo, van a permitir ofrecer a los profesionales sanitarios una serie de productos y servicios.
En el marco del Plan de Calidad, y a través de la Agencia de Calidad del SNS, se ponen en marcha líneas de actuación. Estas han contado con la participación de las agencias y unidades de evaluación de tecnologías sanitarias y otros agentes, con los cuales ha sido posible trabajar, y ofrecer así, productos y servicios de calidad al conjunto de profesionales del SNS.

Con la participación de la red de colaboración de GuíaSalud y con los distintos órganos de dirección y gestión, se trabaja en el desarrollo de las distintas áreas de trabajo que abarcan tanto el entorno de las GPC, con importantes logros a través del tiempo, como el trabajo sobre otros productos, que no siendo GPC se desarrollan con una metodología de trabajo basada en la evidencia científica. Dada la gran diversidad de productos que pueden tener cabida en este grupo, se trabaja en distintas direcciones, por un lado en la propuesta de diversas definiciones para productos que van a tener cabida en la cartera de servicios de GuíaSalud y, por otro lado, en identificar las necesidades que los profesionales sanitarios tienen en cuanto al acceso y uso de productos que faciliten la toma de decisiones basadas en la evidencia científica y cuál o cuáles consideran prioritarios para su desarrollo en el SNS. 


\section{Resultados}

Se ha consensuado y editado un manual metodológico de elaboración de GPC. En breve, se dispondrá de dos manuales más sobre actualización e implementación de GPC.

En noviembre de 2008 se presentan las 8 primeras GPC elaboradas dentro del marco del Plan de Calidad del año 2006. Estas GPC abordan temas de las líneas estratégicas descritas en dicho plan y está previsto que se presenten en breve 13 GPC más. Para ello se ha contado con el trabajo de las agencias y unidades de evaluación de tecnologías sanitarias.

Mediante una segunda vía, se ha desarrollado una GPC sobre reflujo vésicoureteral primario y se viene trabajando en el desarrollo de otra GPC con la participación de numerosas sociedades científicas (SSCC).

En todo este proceso se ha contado con la participación de 477 profesionales del SNS y se ha precisado de la realización de 60 actividades formativas que facilitasen el proceso de elaboración de GPC a los distintos integrantes de los grupos de trabajo.

Con el fin de facilitar el acceso a la información contenida en las GPC, se ha trabajado en la creación de diversas versiones de GPC como son: la versión completa, resumida, herramientas de consul- ta rápida, material para pacientes y material metodológico.

Asimismo, y atendiendo al número de grupos elaboradores, se ha trabajado en la creación de manuales de estilo, tanto para las versiones completa y resumida como en formato html, a los que se puede acceder a través de la siguiente URL: www.guiasalud.es/egpc/index.html. Los profesionales y usuarios de GuíaSalud encontrarán también las GPC en inglés, así como las versiones de consulta rápida en gallego, catalán y euskera.

El profesional sanitario que precise disponer de la información contenida en las GPC elaboradas y publicadas en el marco del Plan de Calidad del SNS a través de un medio distinto como la PDA puede hacerlo a través de la siguiente URL: www.guiasalud.es/PDA/index.htm.

El Comité Científico, la red de colaboración y la Secretaría de GuíaSalud vienen trabajando en el desarrollo de otros productos basados en la evidencia científica. Se ha dotado a la Secretaría de una definición de "pregunta clínica" y de unos criterios para su inclusión en GuíaSalud. A partir de aquí, se identificaron grupos con una trayectoria temporal destacable en su elaboración y se inicia un proceso con el fin de elaborar una documentación consensuada entre todos, que estandarice su elaboración, dada la 
variabilidad registrada tras el estudio llevado a cabo desde GuíaSalud, tanto entre grupos elaboradores en lengua castellana como entre grupos elaboradores en lengua inglesa. De las fuentes elaboradoras de preguntas clínicas en lengua castellana, se obtuvo una muestra de preguntas clínicas que fueron analizadas a través de los criterios propuestos por el Comité Científico de GuíaSalud. Ninguna de las fuentes identificadas y consultadas presentaba una declaración de intereses de las personas encargadas de elaborar las preguntas clínicas. Las fuentes en lengua inglesa fueron valoradas mediante una parrilla de ítems que analizaban tanto la calidad de la información como la calidad formal de la fuente en cuestión.

Dada la relevancia que los profesionales sanitarios han dado a las preguntas clínicas en el estudio cualitativo llevado a cabo y, que va a ser de utilidad para proponer las líneas estratégicas, para la elaboración de otros productos basados en la evidencia, se trabajó en la creación de un buscador de preguntas clínicas en lengua castellana y otro en lengua inglesa.

Para hacer más visible y accesible el trabajo llevado a cabo desde GuíaSalud y todo su entorno, se viene desarrollando una web 2.0 que estará en funcionamiento en breve, donde el usuario podrá registrarse y acceder a múltiples aplica- ciones, desde la herramienta informática para la elaboración de GPC a wikis, blogs, foros, etc....

La Secretaría de GuíaSalud-Biblioteca de GPC del SNS ha conseguido reunir a un grupo multidisciplinar de profesionales en torno a una metodología de trabajo basada en la identificación de la mejor evidencia científica disponible y con aptitudes para el desempeño de su labor con grupos diversos de trabajo.

\section{Conclusiones}

GuíaSalud juega un papel destacado y se le reconoce como tal, en el desarrollo de instrumentos que permitan estandarizar la elaboración de productos, algunos de los cuales venían ofreciéndose con metodologías de elaboración dispares y con estilos de presentación variados.

Con un esfuerzo añadido, GuíaSalud y las agencias y unidades de evaluación de tecnologías sanitarias, en su estrategia de máxima difusión de los productos elaborados, materializan la traducción a lengua inglesa de las GPC elaboradas en el marco del Plan de Calidad.

GuíaSalud ha conseguido, con la ayuda de las agencias y unidades de evaluación de tecnologías sanitarias y la Agencia de Calidad del SNS, coordinar la labor de elaboración de GPC de más de 20 grupos multidisciplinares. 
GuíaSalud provee de la asesoría pertinente y adaptada a las necesidades que, dentro del programa de elaboración de GPC, solicitan grupos, fundamentalmente SSCC y grupos de trabajo concienciados con la necesidad de reducir la variabilidad en la práctica clínica.

GuíaSalud pretende perfilarse como un recurso, puesto a disposición de los profesionales sanitarios del SNS, independiente y con un abanico de productos elaborados con una metodología sistemática y explícita. Las preguntas clínicas constituyen un ejemplo de esta línea de trabajo, fundamentada en la participación de la red de colaboración de GuíaSalud y de todas aquellas instituciones y entidades interesadas en desarrollar productos, basados en la evidencia científi$\mathrm{ca}$, que faciliten la toma de decisiones de los profesionales sanitarios.

El registro como usuarios de la web de GuíaSalud va a permitir conocer los perfiles de los usuarios, sus preferencias y demandas, con el fin de ofrecer una variedad de productos y recursos adaptados, así como ofrecer agilidad en el proceso de actualización de contenidos e inclusión de aquellos elementos, resultado de la innovación tecnológica que permitan y faciliten el acceso a la información, a la discusión en grupos especializados, e incluso a la propia elaboración de productos con nociones básicas sobre metodología.

\section{Bibliografía}

1. GuíaSalud. Guías de práctica clínica en el Sistema Nacional de Salud [sede Web]. Zaragoza: GuíaSalud; 2005 [consultado el 13/09/2009]. Disponible en www.guiasalud.es [actualizada el 11/09/2009].
2. Grupo de trabajo sobre GPC. Elaboración de guías de práctica clínica en el Sistema Nacional de Salud. Manual metodológico. Madrid: Plan Nacional para el SNS del MSC. Instituto Aragonés de Ciencias de la Salud-I+CS; 2007. Guías de práctica clínica en el SNS: I+CS N. ${ }^{\circ}$ 2006/0l. 